Published in final edited form as:

Exerc Sport Sci Rev. 2018 October ; 46(4): 205-214. doi:10.1249/JES.0000000000000158.

\title{
The Renin-Angiotensin System and Skeletal Muscle
}

\author{
Scott K. Powers ${ }^{1}$, Aaron Morton ${ }^{1}$, Hayden Hyatt ${ }^{1}$, Matthew J. Hinkley ${ }^{1}$ \\ ${ }^{1}$ Department of Applied Physiology and Kinesiology, University of Florida, Gainesville, Florida
}

\begin{abstract}
The renin-angiotensin system (RAS) plays a key role in the control of blood pressure and fluid homeostasis. Emerging evidence also reveals that hyperactivity of the RAS contributes to skeletal muscle wasting. This review discusses the key role that the RAS plays in skeletal muscle wasting due to congestive heart failure, chronic kidney disease, and ventilator-induced diaphragmatic wasting.
\end{abstract}

\section{Summary for TOC}

The renin-angiotensin system plays a key role in skeletal muscle wasting due to heart failure, chronic kidney disease, and prolonged mechanical ventilation.

\section{Keywords}

muscle atrophy; diaphragm; ventilator-induced diaphragm dysfunction; weaning; reactive oxygen species

\section{INTRODUCTION}

The role that the renin-angiotensin system (RAS) plays in systems physiology has been investigated for over a century. Indeed, the enzyme renin and its production in the kidney was discovered in 1898 (1). Since this original finding, the RAS has been widely studied for its responsibility in the control of blood pressure, fluid homeostasis, and electrolyte balance (2). While the canonical role of the RAS is focused around these systems, growing evidence also implicates aberrant RAS signaling in the pathogenesis of obesity, insulin resistance, and several conditions that promote skeletal muscle atrophy (2). This review focuses on the emerging evidence that signaling through the RAS contributes to skeletal muscle atrophy in response to chronic disease (e.g., congestive heart failure or chronic kidney disease) and prolonged mechanical ventilation. Further, we discuss several therapeutic approaches to prevent RAS-signaling induced muscle wasting including the potential protective benefits of endurance exercise training.

Corresponding author: Scott K. Powers, Department of Applied Physiology and Kinesiology, University of Florida, Gainesville, FL 32611-8208, spowers@hhp.ufl.edu; Telephone: 352-294-1713.

The authors do not have any conflicts of interest to report in regard to this work. 


\section{OVERVIEW OF THE RENIN-ANGIOTENSIN SYSTEM}

The RAS involves several peptide hormones that exert their influence on numerous physiological functions by binding to a family of RAS receptors. The action of the RAS involves two distinct signaling corridors labeled as the: 1) classical pathway; and 2) nonclassical pathway. This segment discusses both the biochemical pathways responsible for the formation of RAS hormones and the key receptors involved in RAS signaling.

\section{Formation of RAS hormones}

The reactions leading to the production of biologically active RAS hormones begins with the cleavage of angiotensinogen by the protease renin to form angiotensin I (AngI) (Figure 1). AngI is then cleaved by angiotensin converting enzyme (ACE) to produce angiotensin-II (AngII) which is the primary hormone involved in the classical RAS pathway. Conversely, the principal hormone involved in the non-classical RAS pathway is angiotensin 1-7 (Ang17); Ang1-7 can be produced by three different reactions. First, Ang1-7 can be formed from AngII by the catalytic activity of angiotensin converting enzyme 2 (ACE2) (Figure 1). Second, Ang1-7 can be generated from angiotensin 1-9 (Ang1-9) via the enzymatic activity of ACE. Finally, Ang1-7 can also be formed by the conversion of AngI into Ang1-7 by action of neutral endopeptidase (NEP) (Figure 1). Importantly, the signaling actions of AngII and Ang1-7 are antagonistic and this important issue will be discussed in a later section.

\section{Overview of RAS receptors}

While several putative angiotensin receptors have been identified, the three most investigated membrane receptors for RAS hormone peptides are three separate G-protein-coupled receptors: 1) angiotensin II type 1 receptor (AT1R); 2) angiotensin II type 2 receptor (AT2R); and the 3) MAS receptor (MASR). These RAS receptors are expressed in many tissues including the heart, adrenal cortex, adrenal medulla, kidney, brain, vasculature, smooth muscle, and skeletal muscle fibers (3-5). Although RAS receptors are expressed ubiquitously throughout the body, the abundance and biological actions of RAS receptors differ across cell types. This review will focus on the abundance and actions of RAS receptors in skeletal muscle fibers with a specific focus on the roles that the AT1R and MASR play in the classical and non-classical pathways of RAS signaling, respectively.

\section{CLASSICAL RAS PATHWAY IN SKELETAL MUSCLE: COMPONENTS AND SIGNALING}

Activation of the classical RAS signaling pathway begins with the binding of the RAS hormone AngII to the AT1R on the cell membrane. This can occur via both endocrine and autocrine signaling. Endocrine signaling occurs when circulating AngII binds to AT1R on cell membranes (Figure 1). Further, a local (i.e., autocrine) RAS system may exist in several tissues including cardiac and skeletal muscle myocytes (6-8). Autocrine signaling occurs when AngII produced in the cell is released into the interstitial space where it binds to the AT1R. The potential significance of autocrine signaling in skeletal muscle has been recently reviewed and will not be addressed here (9). Moreover, note that a functional RAS system in 
mitochondria has also been discovered and this important finding opens a new field of research involving AngII-mediated intracrine signaling (10). Finally, AT1Rs can also be activated, independent of ligand binding, through mechanical stress applied to the receptor (11).

Activation of the AT1R due to AngII binding or mechanical activation of the receptor results in G-protein coupled signaling that promotes activation of numerous cytosolic signaling pathways. Due to space limitations, only a short summary of these signaling pathways will be provided here and the reader is referred elsewhere for reviews on this topic $(12,13)$. Briefly, activation of the AT1R initiates receptor interaction with several heterotrimeric Gproteins that transduce signals to several downstream second messengers including the mitogen-activated protein kinase (MAPK) family (e.g., p38 MAPK and extracellular signal regulated kinase 1/2 (ERK1/2)), calcium dependent protein kinase $\mathrm{C}$ (PKC), phospholipase $\mathrm{C}$ (PLC), Janus kinase (JAK), and signal transducers and activators of transcription (STAT) (12). Moreover, activation of AT1R results in activation of NADPH oxidase and increased levels of free calcium in the cell that can influence numerous cellular signaling events (14) (Figure 1).

A key component of AT1R signaling that likely contributes to muscle wasting is the production of reactive oxygen species (ROS). Indeed, oxidative stress plays an important role in promoting skeletal muscle atrophy by accelerating proteolysis and depressing protein synthesis (15-20). Activation of the classical RAS axis promotes oxidant production, at least in part, because AT1R signaling activates the predominant NADPH oxidase isoform in skeletal muscle (NOX2) resulting in the production of superoxide radicals $(14,21)$. Further, cross-talk exists between NOX2 and mitochondria whereby activation of NOX2 results in increased ROS production in mitochondria (Figure 1). The mechanisms responsible for the redox cross-talk between NOX 2 and mitochondria has been recently reviewed and therefore, only a brief summary is provided here (22).

At least four mechanisms can explain why activation of NOX2 results in increased mitochondrial production of ROS. First, NOX2 generation of superoxide radicals and the subsequent production of hydrogen peroxide $\left(\mathrm{H}_{2} \mathrm{O}_{2}\right)$ can oxidize proteins in the electron transport chain complexes leading to uncoupling of mitochondria respiration and increased production of superoxide radicals (23). Second, increased NOX2 ROS production results in oxidation of mitochondrial glutathione which diminishes the ability of glutathione peroxidase to remove $\mathrm{H}_{2} \mathrm{O}_{2}$ (23). Thirdly, depletion of mitochondrial glutathione can also increase the production of $\mathrm{H}_{2} \mathrm{O}_{2}$ via the pyruvate dehydrogenase complex (24). Finally, NOX2-derived ROS production can promote calcium release from the sarcoplasmic reticulum resulting in increased cytosolic levels of free calcium (25); this increased calcium leads to mitochondrial calcium overload and augmented production of ROS in the mitochondrion (26).

Notably, AT1R signaling reactions are tissue specific. Indeed, knockout of the AT1R receptor in specific tissues reveals that AT1R second messenger signaling pathways differ across cell types and therefore, it is important to investigate AT1R signaling in a tissue specific manner (reviewed in (12)). This report will specifically focus on the role that 
classical RAS signaling plays in promoting atrophy in skeletal muscle fibers. In particular, we will address the role that RAS signaling plays in promoting skeletal muscle wasting due to chronic disease (e.g., congestive heart failure or end-stage renal disease) and we will also highlight the recent discovery that RAS signaling is a significant contributor to a unique type of disuse muscle atrophy (i.e., ventilator-induced diaphragm atrophy).

\section{NON-CLASSICAL RAS PATHWAY IN SKELETAL MUSCLE: COMPONENTS AND SIGNALING}

The non-classical RAS axis exists in many tissues and interestingly, signaling through this pathway antagonizes signaling through the classical RAS axis. As mentioned earlier, Ang17 is the principal hormone involved in the non-classical RAS pathway and the cellular effects of this hormone are mediated via the MASR (reviewed in $(9,27)$ ) (Figure 1). The signaling events associated with MASR activation remains an active area of study and a thorough discussion of this topic exceeds the scope of this review. Nonetheless, a short introduction to MASR signaling is warranted. Briefly, evidence reveals that MASR activation via Ang1-7 results in direct inhibition of AT1R activation and its down-stream effects (Figure 1) (28, 29). For example, activation of AT1R results in cellular increases in MAPKs; in contrast, Ang1-7 binding to the MASR results in a significantly lower activity of both ERK 1/2 and p38 and increased phosphorylation of protein kinase B (AKT) $(30,31)$. Importantly, activation of the MASR can also prevent AT1R-mediated activation of NADPH oxidase (32). The potential therapeutic benefit of activating the non-classical RAS pathway to oppose the detrimental effects of AT1R activation on skeletal muscle health will be addressed later.

\section{CLASSICAL RAS SIGNALING AND SKELETAL MUSCLE ATROPHY}

Classical RAS signaling promotes skeletal muscle atrophy in several chronic diseases and in patients exposed to prolonged mechanical ventilation. The RAS-mediated skeletal muscle atrophy that ensues during chronic diseases occurs in all skeletal muscles (i.e., locomotor and respiratory muscles) and this form of RAS-mediated atrophy is triggered by AngII induced activation of AT1Rs. In contrast, the rapid RAS-mediated wasting of respiratory muscles that occurs during prolonged mechanical ventilation is independent of elevated AngII levels secondary to chronic disease and is caused by mechanical activation of the AT1R. Therefore, because of these differences in how the AT1R is activated, we will discuss RAS-mediated atrophy of skeletal muscles due to chronic disease and prolonged ventilation separately.

\section{Chronic disease, classical RAS signaling, and muscle atrophy}

Skeletal muscle wasting due to chronic disease (i.e., cachexia) is a serious complication associated with several pathologies including congestive heart failure (CHF) and chronic kidney disease (CKD). Progressive cachexia results in skeletal muscle weakness and impairs quality of life. Importantly, cachexia can independently exacerbate the clinical outcomes of disease in both CHF and CKD patients (13). Therefore, developing therapeutic strategies to prevent disease-related skeletal muscle wasting is important. 
Although several factors could contribute to the development of cachexia in both CHF and CKD, growing evidence suggests that high levels of circulating AngII plays an important role in the muscle wasting associated with both of these diseases. Indeed, patients with CHF or CKD often exhibit plasma AngII levels that are 2-5 times greater than healthy people (13). The mechanisms responsible for increased circulating AngII have been reviewed in detail elsewhere $(33,34)$ and will not be addressed here. This disease-related increase in circulating AngII is significant because pre-clinical studies confirm that elevated circulating AngII promotes significant atrophy in limb muscles in animals free from kidney or heart disease(21, 35-38). Essential to the discussion of muscle wasting, we will introduce the factors that regulate muscle mass and discuss the signaling pathways that connect high levels of circulating AngII to skeletal muscle atrophy in the ensuing segments.

\section{Regulation of skeletal muscle mass-big picture}

Skeletal muscle is the largest organ in the body and comprises $\sim 40 \%$ of total body weight. The maintenance of skeletal muscle mass is dependent on the balance between the rates of protein synthesis and degradation (Figure 2). In chronic diseases that promote high circulating levels of AngII (e.g., CHF or CKD), skeletal muscle proteostasis is disturbed and fiber atrophy occurs due to both decreased muscle protein synthesis and accelerated proteolysis (reviewed in (13)). For example, exposure of murine myotubes to AngII decreases protein synthesis by 40-50\% (39). Studies also reveal that high levels of circulating AngII is associated with large increases in proteolysis due to activation of the major proteolytic systems in skeletal muscle fibers (e.g., ubiquitin-proteasome system, caspase-3, calpain)(37).

Numerous studies have investigated the signaling events that connect AngII to decreases in protein synthesis and increases in protease activation? In theory, AngII signaling could negatively impact skeletal muscle protein balance by direct or indirect actions that influence proteostasis in the muscle. In this regard, the simplest explanation for AngII-induced skeletal muscle atrophy is the direct action of AngII on AT1R's expressed on the surface of skeletal muscle fibers. In contrast, it is also possible that circulating AngII can indirectly impact skeletal muscle health by AT1R signaling in other tissues resulting in increased circulating hormones and/or cytokines that negatively impact skeletal muscle fiber size.

\section{Indirect effects of Angll signaling on skeletal muscle protein synthesis and proteolysis}

High levels of circulating AngII can promote skeletal muscle atrophy by indirect actions of AngII on other tissues (reviewed in (13)). Specifically, high circulating levels of AngII has been shown to increase plasma levels of glucocorticoids, interleukin 6 (IL6), and serum amyloid A (SAA) $(35,40)$; collectively, or independently, these molecules can promote skeletal muscle atrophy independent of AT1R activation on muscle fibers $(41,42)$. Further, high circulating AngII levels in rats is associated with decreases in both circulating and muscle fiber levels of the anabolic protein insulin-like growth factor-1 (IGF-1) $(35,36)$.

High plasma levels of glucocorticoids promote skeletal muscle atrophy by increasing protein degradation and decreasing protein synthesis in muscle fibers. This glucocorticoid-mediated skeletal muscle wasting occurs via activation of glucocorticoid receptor signaling that 
inhibits activation of the Akt (protein kinase B)/ mammalian/mechanistic target of rapamycin (Akt/mTOR) pathway resulting in depressed protein synthesis and increased proteolysis via activation of both the ubiquitin-proteasome system and autophagy (reviewed in (43)). Specifically, glucocorticoid binding to the glucocorticoid receptor increases the expression of several target genes including Regulated in Development and DNA Damage 1 (REDD1) and Kruppel-like factor 15 (KLF15). Elevated REDD1 expression inhibits mTOR signaling by increasing the activity of molecules (i.e., tuberous sclerosis complex $1 / 2$ ) that inhibit mTOR activation(44). KLF15 promotes muscle protein breakdown via transcriptional regulation of the Forkhead Box-O (FoxO) transcription factors leading to increased expression of muscle specific components of the ubiquitin-proteasome system of proteolysis (i.e., atrogin-1 and MuRF1) (45). Direct evidence to support the notion that high circulating glucocorticoids contribute to AngII-mediated muscle atrophy is supported by the discovery that pharmacological blockade of the glucocorticoid receptor partially blunts muscle atrophy induced by high circulating levels of AngII (37). This finding suggests that AngII can indirectly promote muscle atrophy, at least in part, via glucocorticoid signaling.

High circulating glucocorticoids are also associated with an increased expression of myostatin. Myostatin is a member of the transforming growth factor beta (TGF $\beta$ ) family and is expressed and secreted by skeletal muscle; importantly, myostatin functions as a negative regulator of muscle fiber size (reviewed in (46)). Myostatin signaling in muscle occurs via binding to the transmembrane activin A receptor; activation of this receptor promotes an increase in the Smad2 and Smad3 transcription factors which mediate myostatin's effects on muscle mass (47). Although a detailed understanding of the mechanism(s) of Smaddependent muscle atrophy remain unclear, it appears that increased expression of myostatin in skeletal muscle promotes muscle atrophy, in part, by blocking Akt/mTOR signaling and increasing the expression of muscle atrophy genes (atrogenes) such as atrogin-1 (47).

As mentioned earlier, plasma levels of IL6 and SAA are elevated in mice infused with AngII and notably, IL6 and SAA act synergistically to increase the expression of suppressor of cytokine signaling 3 (SOCS-3) in muscle (40). Increased expression of SOCS-3 downregulates insulin receptor substrate-1 (IRS-1) resulting in decreased IGF-1 signaling; this leads to depression of the Akt/mTOR signaling pathway that eventually increases proteolysis and decreases protein synthesis leading to muscle atrophy (40). Moreover, infusion of AngII decreases both plasma and muscle levels of IGF-1. This is significant because a decrease in the IGF-1/PI3K/Akt pathway can lead to muscle atrophy (48). Evidence that depressed IGF-1 signaling contributes to AngII-mediated atrophy is provided from the observation that overexpression of IGF-1 in muscle fibers protects limb muscles against AngII-induced muscle wasting (37).

Finally, decreased food intake is often a problem for patients suffering from CHF or CKD. Obviously, inadequate intake of calories and protein can result in skeletal muscle wasting. Appetite is regulated by the interaction of several complex influences including circulating factors secreted from peripheral tissues (e.g., adipose tissue and gastrointestinal tract) and hypothalamic neuropeptides (reviewed in (13)). In this regard, high circulating levels of AngII depresses appetite due to the effects of AngII on hypothalamic neuropeptides that regulate food intake (36). While it is apparent that high plasma levels of AngII depresses 
appetite, it remains unclear as to whether AngII exerts its influence on the hypothalamus directly (i.e., AngII crossing the blood-brain barrier) or indirectly via a mechanism that translates elevated plasma AngII into signals that influence the synthesis of hypothalamic neuropeptides that regulate satiety $(49,50)$.

In summary, both CHF and CKF result in high plasma levels of AngII that can indirectly promote skeletal muscle atrophy independent of AT1R activation on the skeletal muscle membrane. A synopsis of the molecules and signaling pathways involved in the indirect effects of AngII on muscle atrophy are provided in Figure 3.

\section{Direct effects of Angll/AT1R signaling on skeletal muscle protein synthesis and proteolysis}

In addition to the "indirect" actions of circulating AngII, AngII can also exert a catabolic influence on skeletal muscle fibers by direct activation of AT1Rs on the muscle fiber membrane. As mentioned earlier, AT1R signaling activates NOX2 resulting in the production of ROS. The mechanism(s) responsible for this AT1R-mediated activation of NOX2 remains a topic of debate. Nonetheless, it appears likely that AngII regulates NOX2 activity via activation of PKC and/or activation of the tyrosine kinase Src; active PKC and Src are both capable of phosphorylating the p47phox subunit of NOX2 which is a required step for the correct assembly and activation of this oxidase (reviewed in (22)) (Figure 4).

Moreover, it appears that cross-talk exists between NOX2 and mitochondria whereby NOX2 production of ROS promotes an increase in mitochondrial ROS production; this increase in mitochondrial ROS emission has been reported to further activate NOX2 resulting in the production of additional ROS (22). Collectively, this AT1R-mediated increase in ROS production in muscle fibers results in redox disturbances (i.e., oxidative stress) in the muscle fiber leading to accelerated protein breakdown (51-56) and depressed protein synthesis (57) (Figure 4). Indeed, direct evidence exists to directly link NOX2 activation to AngII-mediated muscle atrophy as, compared to controls, NOX2 knockout animals experience less atrophy following infusion of AngII (21).

As introduced above, a direct causal link exists between oxidative stress and skeletal muscle wasting. Indeed, oxidative stress can activate all four major proteolytic systems in skeletal muscles (reviewed in (17)). Specifically, oxidative stress can accelerate proteolysis by: 1) increasing gene expression and elevating the abundance of proteolytic proteins; 2) allosteric activation of key proteases (e.g., calpain and caspase-3); and 3) oxidizing muscle proteins and increasing their susceptibility to proteolytic breakdown (58). Further, oxidative stress has shown to decrease protein synthesis in skeletal muscles by depressing Akt/mTOR signaling (57).

Despite the evidence of direct binding of AngII on AT1R on skeletal muscle fibers, debate exists over the relative abundance of AT1Rs in skeletal muscle fibers. For example, it has been suggested that only small numbers of AT1Rs exist in mouse skeletal muscle (40). In contrast, opposing evidence suggests that AT1Rs are prominent in rat limb muscle (5), rat diaphragm muscle fibers (4), and murine myoblasts (59). Importantly, both AT1R protein and mRNA has been identified in human limb muscle (i.e., vastus lateralis muscle) in both healthy individuals and patients with congestive heart failure or muscular dystrophy $(60,61)$. 
Therefore, it appears that AT1Rs exist in skeletal muscle and this supports the prediction that AngII exerts a catabolic influence on skeletal muscle fibers by direct activation of AT1R's on the muscle fiber membrane.

To summarize, if a sufficient number of AT1Rs exist on the sarcolemma of a muscle fiber, high circulating levels of AngII trigger AT1R signaling in the muscle fiber and subsequently activate NOX2. Activation of NOX2 in muscle fibers results in the production of ROS and oxidative stress leading to muscle atrophy due to both increased proteolysis and decreased protein synthesis.

\section{Therapeutic approaches to prevent RAS-induced atrophy in limb skeletal muscle}

Several different pharmacological approaches have the potential to protect against AngIIinduced muscle wasting. An obvious approach to lowering circulating levels of AngII is to block the conversion of AngI to AngII using an ACE inhibitor. In this regard, studies reveal that treatment of CHD or CKD patients with ACE inhibitors decreases the disease-related decline in limb muscle strength and improves respiratory muscle strength $(62,63)$.

Pharmacological blockade of AT1Rs is another logical approach to prevent activation of AngII-induced RAS signaling in CHD or CKD patients. Similar to the clinical trials using ACE inhibitors, preclinical studies demonstrate that pharmacological blockade of AT1Rs protect against limb muscle atrophy in a rat model of experimental heart failure (64). Although no published reports in humans currently exist, it is feasible that AT1R blockade can protect against CHF or CKD-induced muscle wasting in humans.

Another pharmacological approach that could protect skeletal muscles against AngIIinduced muscle wasting involves activation of the non-classical pathway of RAS signaling. Recall that MASR activation via Ang1-7 results in direct inhibition of AT1R signaling (28). Further, numerous studies conclude that MASR's are abundant in rodent skeletal muscle fibers (65-69), Importantly, preclinical studies reveal that infusion of Ang1-7 in rats activates MASR signaling and protects skeletal muscle against AngII-induced muscle atrophy $(66,70)$. Nonetheless, it is currently unknown if MASR's exist in human skeletal muscle fibers and it is also undetermined if infusion of Ang1-7 will protect against muscle wasting in humans suffering from CHF or CKD.

Finally, endurance exercise training increases exercise tolerance and improves the quality of life in CHF patients (71). The mechanism(s) responsible for this exercise-induced effect is uncertain but could be linked to exercise-induced changes in RAS signaling. For example, exercise training decreases both ACE and AngII concentrations in the rat heart and exercise also increases ACE2 and Ang1-7 concentrations in cardiac myocytes (72). Moreover, endurance exercise training increases the ratio of Ang1-7/AngII in limb muscle fibers of rats with CHF (73). Together, this evidence suggests that endurance exercise training promotes a shift in relative RAS signaling in skeletal muscles away from the classical pathway toward the non-classical pathway. Future experiments are required to confirm that endurance exercise training promotes changes in RAS signaling and protects against RAS-induced wasting in human skeletal muscles. 


\section{RAS signaling and diaphragmatic atrophy during prolonged mechanical ventilation}

This segment highlights the exciting discovery that RAS signaling is required for the rapid atrophy that occurs in inspiratory muscles during prolonged mechanical ventilation (MV). For those readers not familiar with MV-induced inspiratory muscle weakness, MV is a clinical intervention used to maintain pulmonary gas exchange in patients unable to do so with spontaneous breathing. Conventional applications for MV include surgery, critical illness, and drug overdose. Although MV is often a life-saving measure, an unintended consequence of prolonged MV is the rapid development of weakness in the primary inspiratory muscle, the diaphragm. Indeed, in both humans and other mammals, MVinduced diaphragmatic weakness occurs within the first 12-24 hours of MV and is due to both diaphragm fiber atrophy and contractile dysfunction (74-81). Figure 5 illustrates the MV-induced diaphragmatic atrophy in humans during ventilator support. Importantly, notice the rapid rate of diaphragmatic atrophy after the initiation of MV; this magnitude of fiber atrophy in limb muscle would require 5 days or more of muscle inactivity (e.g., hindlimb unloading) (82).

Because the diaphragm is the principal inspiratory muscle and essential to maintain blood gas homeostasis, MV-induced diaphragmatic atrophy is an important clinical problem because inspiratory muscle weakness is a major risk factor for problems in "weaning" patients from the ventilator (83). Failure to wean patients from the ventilator results in extended hospitalization and a significant increase in patient morbidity and mortality (84). Therefore, prevention of MV-induced diaphragmatic weakness is important. Developing a therapeutic strategy to prevent ventilator-induced diaphragm weakness requires a thorough understanding of the mechanism(s) responsible for this problem. The next segments provide details about MV-induced diaphragmatic weakness and examine the link between RAS signaling and MV-induced diaphragmatic weakness.

During full support MV, the ventilator performs all the work of breathing and the diaphragm remains inactive. Hence, ventilator-induced diaphragm inactivity is a unique form of disuse muscle atrophy. Indeed, rodent models of MV reveal that as few as 12 hours of MV results in a $10-15 \%$ reduction in diaphragmatic fiber cross sectional area (reviewed in (85)). This rapid rate of diaphragmatic atrophy is remarkable given that it would require 5-7 days to achieve a similar level of atrophy in rodent limb muscles exposed to inactivity (82). The mechanism(s) responsible for this swift rate of atrophy have been the focus of numerous studies and similar to inactivity-induced atrophy in limb muscles, MV-induced diaphragmatic atrophy occurs due to both accelerated proteolysis and a decreased rate of protein synthesis $(55-57,78,81,86,87)$. The key question becomes, what triggers this rapid rate of atrophy? In this regard, increases in ROS production in diaphragm fibers play a required role in the rapid development of MV-induced proteolysis and depressed protein synthesis in the diaphragm $(51,53,56,88)$. The cell signaling pathways that trigger the MVinduced increase in ROS production in the diaphragm remains an active area of research; however, a recent study reveals that the RAS plays a key role in this process.

Prolonged MV in rodents results in increased plasma levels of AngII, thus serving as a potential mechanism to promote diaphragmatic atrophy (4). However, treatment of animals with an ACE inhibitor to prevent the MV-induced increase in AngII does not protect against 
MV-induced diaphragmatic atrophy (4). In contrast, treatment of animals with the AT1R blocker losartan attenuates MV-induced diaphragmatic atrophy (4). These results indicate that high levels of circulating AngII are not required for the development of MV-induced diaphragmatic atrophy and raise the question, "why does an AT1R blockade protect against $\mathrm{MV}$-induced diaphragmatic atrophy if prevention of the increase in its activating ligand alone does not provide protection? “

At least two explanations can explain why losartan protects against MV-induced diaphragmatic atrophy. First, losartan could shield against MV-induced diaphragmatic atrophy via an off-target effect of the drug that is not directly linked to AT1R signaling. Alternatively, losartan can protect against MV-induced diaphragmatic atrophy by blocking mechanical activation of the AT1R.

Given that oxidative stress is a required up-stream signal to promote MV-induced diaphragmatic atrophy, a possible off-target effect is that losartan acts as an antioxidant to prevent MV-induced oxidative stress in diaphragm fibers. In this regard, the chemical structure of losartan (i.e., hydroxyl functional group located on a phenolic ring) suggests that losartan is a radical scavenger and therefore, capable of protecting against MV-induced oxidative stress in the diaphragm. Nonetheless, at the concentration used to block AT1R signaling in animals, losartan does not function as an antioxidant (4). Therefore, by elimination, it appears that losartan protects against VIDD by preventing activation of the AT1R.

As discussed previously, debate exists as to whether AngII-induced skeletal muscle atrophy is due to the direct effects of AngII on the muscle fiber or due to systemic effects of AngII resulting in increased levels of circulating glucocorticoids or IL-6, both of which can promote skeletal muscle atrophy. In the case of MV-induced diaphragm atrophy, plasma levels of IL-6 and glucocorticoids are not increased in rodents during 12 hours of MV (4). Hence, the losartan mediated protection against MV-induced diaphragmatic atrophy appears to be linked to losartan's direct effect on diaphragm fibers via inhibition of mechanical activation of the AT1R. In this regard, the presence of AT1R on the sarcolemma of diaphragm muscle fibers has been confirmed (4). Moreover, preventing the increase in circulating increased plasma levels of AngII does not provide protection against MV-induced diaphragm atrophy. Thus, independent of AngII binding to the AT1R, it appears that prolonged MV results in mechanical activation of $\operatorname{AT} 1 \mathrm{R}(11,89)$.

Mechanical activation of AT1R occurs when tension applied to a cell membrane initiates a counter-clockwise rotation in the configuration of the transmembrane AT1R; this movement of the AT1R acts as a conformational switch that couples mechanical stress-induced activation of AT1R signaling (89). Currently, no evidence exists that spontaneous breathing results in mechanical activation of AT1R on diaphragm fibers. So, "why does prolonged MV promote mechanical activation of AT1R on diaphragm fibers whereas spontaneous breathing does not?" Unfortunately, a definitive answer to this question is not available. Nonetheless, a possible explanation is that the tension applied to the sarcolemma and the AT1R of diaphragm fibers differs between spontaneous breathing and MV. During prolonged MV, diaphragm muscle fibers undergo repetitive, passive length changes (i.e., shortening/ 
lengthening cycles). Interestingly, diaphragm fibers shorten more for a given change in lung volume during MV compared to spontaneous breathing (90). It follows that tension applied to the sarcolemma of diaphragm fibers is greater during MV compared to spontaneous breathing $(91,92)$. Hence, these differences in mechanical stress on diaphragm fibers may explain why prolonged MV results in mechanical activation of the AT1R whereas spontaneous breathing does not; this is a testable hypothesis worthy of additional research.

\section{Therapeutic approaches to protect diaphragm skeletal muscle against MV-induced atrophy}

In contrast to ACE inhibitors, pharmacological blockade of AT1R's protects the rat diaphragm against MV-induced atrophy (4). Although no human data exist, it is feasible that AT1R blockade could protect against MV-induced diaphragm atrophy in humans. This is an exciting area for future work.

Another pharmacological approach that could shield the diaphragm against MV-induced atrophy involves Ang1-7 activation of the MASR resulting in signaling through the nonclassical RAS pathway. In theory, infusion of Ang 1-7 has therapeutic potential for the prevention of MV-induced diaphragmatic atrophy (93).

Finally, while whole body exercise results in adaptations in the active limb muscles, endurance exercise training also results in a "preconditioned" diaphragm muscle that is protected against MV-induced diaphragmatic atrophy (94). As discussed earlier, endurance exercise training has also been shown to alter RAS signaling in both cardiac and limb muscle resulting in an apparent shift in the balance of RAS signaling away from the classical pathway toward the non-classical pathway. It is currently unclear if exercise training results in similar adaptations in the diaphragm and this is an important area for future research.

\section{FUTURE PERSPECTIVES-WHAT WE KNOW AND WHAT WE DON'T KNOW}

This final section summarizes the established concepts that connect RAS signaling to limb muscle wasting in patients with chronic disease (i.e., CHF and CKD) and to diaphragmatic atrophy in individuals exposed to prolonged MV. In hopes of stimulating future research, we will then summarize the unresolved questions linked to RAS signaling and skeletal muscle.

\section{What we know}

Numerous studies confirm that high plasma levels of AngII activates AT1R signaling which promotes the skeletal muscle wasting associated with both CHF and CKD. Indeed, high circulating levels of AngII exhibit significant atrophy in both limb and respiratory muscles due to decreased protein synthesis and increased proteolysis in muscle fibers. Notably, studies have also demonstrated that prevention of AngII signaling via ACE inhibitors or blockade of AT1Rs prevents muscle atrophy resulting from high levels of circulating AngII.

Emerging evidence indicates that prolonged MV results in mechanical activation of AT1Rs in diaphragm muscle and that this AT1R activation plays an important role in MV-induced oxidative stress, protease activation, and atrophy of diaphragm muscle fibers. Importantly, this MV-induced diaphragmatic weakness contributes to difficulties in weaning patients from the ventilator. Preclinical studies reveal that ACE inhibitors do not protect the 
diaphragm against MV-induced diaphragmatic atrophy. In contrast, treatment of animals with AT1R blockers appears to rescue the diaphragm from MV-induced atrophy.

\section{What we don't know}

Numerous unanswered questions remain regarding the mechanisms responsible for AngIIinduced limb muscle wasting. For example, questions remain regarding whether high levels of circulating AngII promotes muscle wasting due to direct or indirect effects on muscle fibers. More specifically, debate continues about the relative abundance of AT1Rs in limb skeletal muscle. On one hand, some studies suggest that AT1Rs are abundant in limb skeletal muscles. In contrast, another camp argues that AT1Rs exist in low relative abundance in skeletal muscle fibers and that AngII-induced muscle wasting occurs due to the indirect effects of AngII binding to AT1Rs on non-muscle tissues with the end-result being increased circulating levels of glucocorticoids and/or cytokines (e.g., IL6) that promote muscle atrophy. Clearly, additional studies are required to resolve this conflict and determine the relative abundance of AT1Rs in skeletal muscles differing in fiber types and resolve the question of whether classical RAS signaling promotes muscle atrophy due to direct or indirect effects on muscle fibers.

Limited information currently exists regarding the impact of regular exercise on RAS signaling in skeletal muscles. Indeed, although endurance exercise training improves exercise tolerance in CHF patients, it is unclear if exercise training has a direct effect on altering RAS signaling in locomotor skeletal muscles in humans.

While evidence indicates that pharmacological blockade of AT1Rs protects the diaphragm against MV-induced atrophy, the mechanisms to explain this defense remain obscure. Clearly, in order to develop an evidence-based therapeutic strategy to protect the diaphragm against MV-induced diaphragmatic atrophy, a more detailed understanding of the molecular events that lead to MV-induced mechanical activation of AT1Rs in the diaphragm is required.

\section{Acknowledgements}

This work was supported by a grant from the National Institutes of Health (NIH R01 AR064189 awarded to SKP.

The author would like to thank Kelli Morton for contributing to figure preparation.

\section{References}

1. Phillips MI, Schmidt-Ott KM. The Discovery of Renin 100 Years Ago. News in physiological sciences : an international journal of physiology produced jointly by the International Union of Physiological Sciences and the American Physiological Society. 1999;14:271-4.

2. Ramalingam L, Menikdiwela K, LeMieux M, et al. The renin angiotensin system, oxidative stress and mitochondrial function in obesity and insulin resistance. Biochimica et biophysica acta. 2017;1863(5):1106-14. [PubMed: 27497523]

3. Allen AM, Zhuo J, Mendelshon F Localization and function of Angiotension At1 receptors. American Journal of Hypertension. 2000;13:31S-8S. [PubMed: 10678286]

4. Kwon OS, Smuder AJ, Wiggs MP, et al. AT1 receptor blocker losartan protects against mechanical ventilation-induced diaphragmatic dysfunction. J Appl Physiol. 2015;119(10):1033-41. [PubMed: 26359481] 
5. Linderman JR, Greene AS. Distribution of angiotensin II receptor expression in the microcirculation of striated muscle. Microcirculation. 2001;8(4):275-81. [PubMed: 11528535]

6. Danser AH, Koning MM, Admiraal PJ, et al. Production of angiotensins I and II at tissue sites in intact pigs. Am J Physiol. 1992;263(2 Pt 2):H429-37. [PubMed: 1510141]

7. Leri A, Claudio PP, Li Q, et al. Stretch-mediated release of angiotensin II induces myocyte apoptosis by activating p53 that enhances the local renin-angiotensin system and decreases the Bcl-2-to-Bax protein ratio in the cell. Journal of Clinical Investigation. 1998;101(7):1326-42. [PubMed: 9525975]

8. Sadoshima J, Xu Y, Slayter HS, Izumo S. Autocrine release of angiotensin II mediates stretchinduced hypertrophy of cardiac myocytes in vitro. Cell. 1993;75(5):977-84. [PubMed: 8252633]

9. Cabello-Verrugio C, Morales MG, Rivera JC, Cabrera D, Simon F. Renin-angiotensin system: an old player with novel functions in skeletal muscle. Medicinal research reviews. 2015;35(3):437-63. [PubMed: 25764065]

10. Abadir PM, Foster DB, Crow M, et al. Identification and characterization of a functional mitochondrial angiotensin system. Proc Natl Acad Sci U S A. 2011;108(36):14849-54. [PubMed: 21852574]

11. Zou Y, Akazawa H, Qin Y, et al. Mechanical stress activates angiotensin II type 1 receptor without the involvement of angiotensin II. Nat Cell Biol. 2004;6(6):499-506. [PubMed: 15146194]

12. Kawai T, Forrester SJ, O'Brien S, Baggett A, Rizzo V, Eguchi S. AT1 receptor signaling pathways in the cardiovascular system. Pharmacol Res. 2017;125:4-13. [PubMed: 28527699]

13. Yoshida T, Tabony AM, Galvez S, et al. Molecular mechanisms and signaling pathways of angiotensin II-induced muscle wasting: potential therapeutic targets for cardiac cachexia. Int J Biochem Cell Biol. 2013;45(10):2322-32. [PubMed: 23769949]

14. Cozzoli A, Liantonio A, Conte E, et al. Angiotensin II modulates mouse skeletal muscle resting conductance to chloride and potassium ions and calcium homeostasis via the AT1 receptor and NADPH oxidase. Am J Physiol Cell Physiol. 2014;307(7):C634-47. [PubMed: 25080489]

15. Powers SK, Kavazis AN, DeRuisseau KC. Mechanisms of disuse muscle atrophy: role of oxidative stress. Am J Physiol Regul Integr Comp Physiol. 2005;288(2):R337-44. [PubMed: 15637170]

16. Powers SK, Kavazis AN, McClung JM. Oxidative stress and disuse muscle atrophy. J Appl Physiol. 2007;102(6):2389-97. [PubMed: 17289908]

17. Powers SK, Morton AB, Ahn B, Smuder AJ. Redox control of skeletal muscle atrophy. Free Radic Biol Med. 2016;98:208-17. [PubMed: 26912035]

18. Powers SK, Smuder AJ, Criswell DS. Mechanistic links between oxidative stress and disuse muscle atrophy. Antioxid Redox Signal. 2011;15(9):2519-28. [PubMed: 21457104]

19. Powers SK, Smuder AJ, Judge AR. Oxidative stress and disuse muscle atrophy: cause or consequence? Curr Opin Clin Nutr Metab Care. 2012;15(3):240-5. [PubMed: 22466926]

20. Powers SK, Wiggs MP, Duarte JA, Zergeroglu AM, Demirel HA. Mitochondrial signaling contributes to disuse muscle atrophy. Am J Physiol Endocrinol Metab. 2012;303(1):E31-9. [PubMed: 22395111]

21. Semprun-Prieto LC, Sukhanov S, Yoshida T, et al. Angiotensin II induced catabolic effect and muscle atrophy are redox dependent. Biochem Biophys Res Commun. 2011;409(2):217-21. [PubMed: 21570954]

22. Ferreira LF, Laitano O. Regulation of NADPH oxidases in skeletal muscle. Free Radic Biol Med. 2016;98:18-28.. [PubMed: 27184955]

23. Murphy MP. How mitochondria produce reactive oxygen species. The Biochemical journal. 2009;417(1):1-13. [PubMed: 19061483]

24. Fisher-Wellman KH, Gilliam LA, Lin CT, Cathey BL, Lark DS, Neufer PD. Mitochondrial glutathione depletion reveals a novel role for the pyruvate dehydrogenase complex as a key $\mathrm{H}_{2} \mathrm{O} 2$ emitting source under conditions of nutrient overload. Free Radic Biol Med. 2013;65:1201-8. [PubMed: 24056031]

25. Santulli G, Xie W, Reiken SR, Marks AR. Mitochondrial calcium overload is a key determinant in heart failure. Proc Natl Acad Sci U S A. 2015;112(36):11389-94. [PubMed: 26217001]

26. Peng TI, Jou MJ. Oxidative stress caused by mitochondrial calcium overload. Annals of the New York Academy of Sciences. 2010;1201:183-8. [PubMed: 20649555] 
27. Karnik SS, Singh KD, Tirupula K, Unal H. Significance of angiotensin 1-7 coupling with MAS1 receptor and other GPCRs to the renin-angiotensin system: IUPHAR Review 22. British journal of pharmacology. 2017;174(9):737-53. [PubMed: 28194766]

28. Kostenis E, Milligan G, Christopoulos A, et al. G-protein-coupled receptor Mas is a physiological antagonist of the angiotensin II type 1 receptor. Circulation. 2005;111(14):1806-13. [PubMed: 15809376]

29. Santos RAS, Sampaio WO, Alzamora AC, et al. The ACE2/Angiotensin-(1-7)/MAS Axis of the Renin-Angiotensin System: Focus on Angiotensin-(1-7). Physiol Rev. 2018;98(1):505-53. [PubMed: 29351514]

30. Munoz MC, Giani JF, Dominici FP. Angiotensin-(1-7) stimulates the phosphorylation of Akt in rat extracardiac tissues in vivo via receptor Mas. Regulatory peptides. 2010;161(1-3):1-7. [PubMed: 20188769]

31. Prestes T, Rocha N, Miranda A, Teixeira A, and e Silva A The anti-inflammatory potential of ACE2/Angiotensin-(1-7)/Mas receptor axis: Evidence from basic and clinical research Current Drug Targets. 2016;17:1-13.

32. Villalobos LA, San Hipolito-Luengo A, Ramos-Gonzalez M, et al. The Angiotensin-(1-7)/Mas Axis Counteracts Angiotensin II-Dependent and -Independent Pro-inflammatory Signaling in Human Vascular Smooth Muscle Cells. Front Pharmacol. 2016;7:482. [PubMed: 28018220]

33. Kobori H, Nangaku M, Navar LG, Nishiyama A. The intrarenal renin-angiotensin system: from physiology to the pathobiology of hypertension and kidney disease. Pharmacol Rev. 2007;59(3): 251-87. [PubMed: 17878513]

34. Zucker IH, Schultz HD, Patel KP, Wang H. Modulation of angiotensin II signaling following exercise training in heart failure. Am J Physiol Heart Circ Physiol. 2015;308(8):H781-91. [PubMed: 25681422]

35. Brink M, Price SR, Chrast J, et al. Angiotensin II induces skeletal muscle wasting through enhanced protein degradation and down-regulates autocrine insulin-like growth factor I. Endocrinology. 2001;142(4):1489-96. [PubMed: 11250929]

36. Brink M, Wellen J, Delafontaine P. Angiotensin II causes weight loss and decreases circulating insulin-like growth factor I in rats through a pressor-independent mechanism. The Journal of clinical investigation. 1996;97(11):2509-16. [PubMed: 8647943]

37. Song YH, Li Y, Du J, Mitch WE, Rosenthal N, Delafontaine P. Muscle-specific expression of IGF-1 blocks angiotensin II-induced skeletal muscle wasting. The Journal of clinical investigation. 2005;115(2):451-8. [PubMed: 15650772]

38. Yoshida T, Galvez S, Tiwari S, et al. Angiotensin II inhibits satellite cell proliferation and prevents skeletal muscle regeneration. J Biol Chem. 2013;288(33):23823-32. [PubMed: 23831688]

39. Russell ST, Sanders PM, Tisdale MJ. Angiotensin II directly inhibits protein synthesis in murine myotubes. Cancer letters. 2006;231(2):290-4. [PubMed: 16399230]

40. Zhang L, Du J, Hu Z, et al. IL-6 and serum amyloid A synergy mediates angiotensin II-induced muscle wasting. J Am Soc Nephrol. 2009;20(3):604-12. [PubMed: 19158350]

41. Goodman MN. Interleukin-6 induces skeletal muscle protein breakdown in rats. Proceedings of the Society for Experimental Biology and Medicine Society for Experimental Biology and Medicine. 1994;205(2):182-5.

42. Wing SS, Goldberg AL. Glucocorticoids activate the ATP-ubiquitin-dependent proteolytic system in skeletal muscle during fasting. Am J Physiol. 1993;264(4 Pt 1):E668-76. [PubMed: 7682781]

43. Schakman O, Kalista S, Barbe C, Loumaye A, Thissen JP. Glucocorticoid-induced skeletal muscle atrophy. Int J Biochem Cell Biol. 2013;45(10):2163-72. [PubMed: 23806868]

44. Shimizu N, Yoshikawa N, Ito N, et al. Crosstalk between glucocorticoid receptor and nutritional sensor mTOR in skeletal muscle. Cell metabolism. 2011;13(2):170-82. [PubMed: 21284984]

45. Waddell DS, Baehr LM, van den Brandt J, et al. The glucocorticoid receptor and FOXO1 synergistically activate the skeletal muscle atrophy-associated MuRF1 gene. Am J Physiol Endocrinol Metab. 2008;295(4):E785-97. [PubMed: 18612045]

46. Bonaldo P, Sandri M. Cellular and molecular mechanisms of muscle atrophy. Disease models \& mechanisms. 2013;6(1):25-39. [PubMed: 23268536] 
47. Sartori R, Milan G, Patron M, et al. Smad2 and 3 transcription factors control muscle mass in adulthood. Am J Physiol Cell Physiol. 2009;296(6):C1248-57. [PubMed: 19357234]

48. Bodine SC, Stitt TN, Gonzalez M, et al. Akt/mTOR pathway is a crucial regulator of skeletal muscle hypertrophy and can prevent muscle atrophy in vivo. Nat Cell Biol. 2001;3(11):1014-9. [PubMed: 11715023]

49. Biancardi VC, Stern JE. Compromised blood-brain barrier permeability: novel mechanism by which circulating angiotensin II signals to sympathoexcitatory centres during hypertension. J Physiol. 2016;594(6):1591-600. [PubMed: 26580484]

50. Paton JF, Wang S, Polson JW, Kasparov S. Signalling across the blood brain barrier by angiotensin II: novel implications for neurogenic hypertension. J Mol Med (Berl). 2008;86(6):705-10. [PubMed: 18443753]

51. Betters JL, Criswell DS, Shanely RA, et al. Trolox attenuates mechanical ventilation-induced diaphragmatic dysfunction and proteolysis. Am J Respir Crit Care Med. 2004;170(11):1179-84. [PubMed: 15374845]

52. Min K, Smuder AJ, Kwon OS, Kavazis AN, Szeto HH, Powers SK. Mitochondrial-targeted antioxidants protect skeletal muscle against immobilization-induced muscle atrophy. J Appl Physiol. 2011;111(5):1459-66. [PubMed: 21817113]

53. Powers SK, Hudson MB, Nelson WB, et al. Mitochondria-targeted antioxidants protect against mechanical ventilation-induced diaphragm weakness. Crit Care Med. 2011;39(7):1749-59. [PubMed: 21460706]

54. Talbert EE, Smuder AJ, Min K, Kwon OS, Szeto HH, Powers SK. Immobilization-induced activation of key proteolytic systems in skeletal muscles is prevented by a mitochondria-targeted antioxidant. J Appl Physiol. 2013;115(4):529-38.. [PubMed: 23766499]

55. Whidden MA, McClung JM, Falk DJ, et al. Xanthine oxidase contributes to mechanical ventilation-induced diaphragmatic oxidative stress and contractile dysfunction. J Appl Physiol. 2009;106(2):385-94. [PubMed: 18974366]

56. Whidden MA, Smuder AJ, Wu M, Hudson MB, Nelson WB, Powers SK. Oxidative stress is required for mechanical ventilation-induced protease activation in the diaphragm. J Appl Physiol. 2010;108(5):1376-82. [PubMed: 20203072]

57. Hudson MB, Smuder AJ, Nelson WB, et al. Partial Support Ventilation and MitochondrialTargeted Antioxidants Protect against Ventilator-Induced Decreases in Diaphragm Muscle Protein Synthesis. PLoS One. 2015;10(9):e0137693. [PubMed: 26361212]

58. Smuder AJ, Kavazis AN, Hudson MB, Nelson WB, Powers SK. Oxidation enhances myofibrillar protein degradation via calpain and caspase-3. Free Radic Biol Med. 2010;49(7):1152-60. [PubMed: 20600829]

59. Johnston AP, Baker J, De Lisio M, Parise G. Skeletal muscle myoblasts possess a stretchresponsive local angiotensin signalling system. J Renin Angiotensin Aldosterone Syst. 2011;12(2): 75-84. [PubMed: 20921089]

60. Malendowicz SL, Ennezat PV, Testa M, et al. Angiotensin II receptor subtypes in the skeletal muscle vasculature of patients with severe congestive heart failure. Circulation. 2000;102(18): 2210-3. [PubMed: 11056094]

61. Sun G, Haginoya K, Dai H, et al. Intramuscular renin-angiotensin system is activated in human muscular dystrophy. Journal of the neurological sciences. 2009;280(1-2):40-8. [PubMed: 19232644]

62. Coirault C, Hagege A, Chemla D, Fratacci MD, Guerot C, Lecarpentier Y. Angiotensin-converting enzyme inhibitor therapy improves respiratory muscle strength in patients with heart failure. Chest. 2001;119(6):1755-60. [PubMed: 11399702]

63. Onder G, Penninx BW, Balkrishnan R, et al. Relation between use of angiotensin-converting enzyme inhibitors and muscle strength and physical function in older women: an observational study. Lancet. 2002;359(9310):926-30. [PubMed: 11918911]

64. Dalla Libera L, Ravara B, Angelini A, et al. Beneficial effects on skeletal muscle of the angiotensin II type 1 receptor blocker irbesartan in experimental heart failure. Circulation. 2001;103(17):2195200. [PubMed: 11331262] 
65. Abrigo J, Simon F, Cabrera D, Cabello-Verrugio C. Angiotensin-(1-7) Prevents Skeletal Muscle Atrophy Induced by Transforming Growth Factor Type Beta (TGF-beta) via Mas Receptor Activation. Cellular physiology and biochemistry : international journal of experimental cellular physiology, biochemistry, and pharmacology. 2016;40(1-2):27-38.

66. Cisternas F, Morales MG, Meneses C, et al. Angiotensin-(1-7) decreases skeletal muscle atrophy induced by angiotensin II through a Mas receptor-dependent mechanism. Clinical science. 2015;128(5):307-19. [PubMed: 25222828]

67. Morales MG, Abrigo J, Acuna MJ, et al. Angiotensin-(1-7) attenuates disuse skeletal muscle atrophy in mice via its receptor, Mas. Disease models \& mechanisms. 2016;9(4):441-9. [PubMed: 26851244]

68. Morales MG, Abrigo J, Meneses C, Cisternas F, Simon F, Cabello-Verrugio C. Expression of the Mas receptor is upregulated in skeletal muscle wasting. Histochemistry and cell biology. 2015;143(2):131-41. [PubMed: 25208653]

69. Morales MG, Olguin H, Di Capua G, Brandan E, Simon F, Cabello-Verrugio C. Endotoxin-induced skeletal muscle wasting is prevented by angiotensin-(1-7) through a p38 MAPK-dependent mechanism. Clinical science. 2015;129(6):461-76. [PubMed: 25989282]

70. Meneses C, Morales MG, Abrigo J, Simon F, Brandan E, Cabello-Verrugio C. The angiotensin-(17)/Mas axis reduces myonuclear apoptosis during recovery from angiotensin II-induced skeletal muscle atrophy in mice. Pflugers Arch. 2015;467(9):1975-84. [PubMed: 25292283]

71. de Mello Franco FG, Santos AC, Rondon MU, et al. Effects of home-based exercise training on neurovascular control in patients with heart failure. Eur J Heart Fail. 2006;8(8):851-5. [PubMed: 16714146]

72. Fernandes T, Hashimoto NY, Magalhaes FC, et al. Aerobic exercise training-induced left ventricular hypertrophy involves regulatory MicroRNAs, decreased angiotensin-converting enzyme-angiotensin ii, and synergistic regulation of angiotensin-converting enzyme 2-angiotensin (1-7). Hypertension. 2011;58(2):182-9. [PubMed: 21709209]

73. Gomes-Santos IL, Fernandes T, Couto GK, et al. Effects of exercise training on circulating and skeletal muscle renin-angiotensin system in chronic heart failure rats. PLoS One. 2014;9(5):e98012. [PubMed: 24859374]

74. Anzueto A, Peters JI, Tobin MJ, et al. Effects of prolonged controlled mechanical ventilation on diaphragmatic function in healthy adult baboons. Crit Care Med. 1997;25(7):1187-90. [PubMed: 9233746]

75. Bruells CS, Smuder AJ, Reiss LK, et al. Negative pressure ventilation and positive pressure ventilation promote comparable levels of ventilator-induced diaphragmatic dysfunction in rats. Anesthesiology. 2013;119(3):652-62. [PubMed: 23838714]

76. Criswell DS, Shanely RA, Betters JJ, et al. Cumulative effects of aging and mechanical ventilation on in vitro diaphragm function. Chest. 2003;124(6):2302-8. [PubMed: 14665514]

77. Gayan-Ramirez G, Testelmans D, Maes K, et al. Intermittent spontaneous breathing protects the rat diaphragm from mechanical ventilation effects. Crit Care Med. 2005;33(12):2804-9. [PubMed: 16352963]

78. Levine S, Biswas C, Dierov J, et al. Increased proteolysis, myosin depletion, and atrophic AKTFOXO signaling in human diaphragm disuse. Am J Respir Crit Care Med. 2011;183(4):483-90. [PubMed: 20833824]

79. Levine S, Nguyen T, Taylor N, et al. Rapid disuse atrophy of diaphragm fibers in mechanically ventilated humans. N Engl J Med. 2008;358(13):1327-35. [PubMed: 18367735]

80. Powers SK, Shanely RA, Coombes JS, et al. Mechanical ventilation results in progressive contractile dysfunction in the diaphragm. J Appl Physiol. 2002;92(5):1851-8. [PubMed: 11960933]

81. Shanely RA, Zergeroglu MA, Lennon SL, et al. Mechanical ventilation-induced diaphragmatic atrophy is associated with oxidative injury and increased proteolytic activity. Am J Respir Crit Care Med. 2002;166(10):1369-74. [PubMed: 12421745]

82. Thomason DB, Biggs RB, Booth FW. Protein metabolism and beta-myosin heavy-chain mRNA in unweighted soleus muscle. Am J Physiol. 1989;257(2 Pt 2):R300-5. [PubMed: 2764153] 
83. Eskandar N, Apostolakos MJ. Weaning from mechanical ventilation. Crit Care Clin. 2007;23(2): 263-74, x. Epub 2007/03/21. [PubMed: 17368170]

84. Esteban A, Alia I, Ibanez J, Benito S, Tobin MJ. Modes of mechanical ventilation and weaning. A national survey of Spanish hospitals. The Spanish Lung Failure Collaborative Group. Chest. 1994;106(4):1188-93. [PubMed: 7924494]

85. Powers SK, Wiggs MP, Sollanek KJ, Smuder AJ. Ventilator-induced diaphragm dysfunction: cause and effect. Am J Physiol Regul Integr Comp Physiol. 2013;305(5):R464-77. [PubMed: 23842681]

86. Shanely RA, Van Gammeren D, Deruisseau KC, et al. Mechanical ventilation depresses protein synthesis in the rat diaphragm. Am J Respir Crit Care Med. 2004;170(9):994-9. [PubMed: 15297271]

87. Smuder AJ, Nelson WB, Hudson MB, Kavazis AN, Powers SK. Inhibition of the ubiquitinproteasome pathway does not protect against ventilator-induced accelerated proteolysis or atrophy in the diaphragm. Anesthesiology. 2014;121(1):115-26. [PubMed: 24681580]

88. Agten A, Maes K, Smuder A, Powers SK, Decramer M, Gayan-Ramirez G. N-Acetylcysteine protects the rat diaphragm from the decreased contractility associated with controlled mechanical ventilation. Crit Care Med. 2011;39(4):777-82. [PubMed: 21242791]

89. Yasuda N, Miura S, Akazawa H, et al. Conformational switch of angiotensin II type 1 receptor underlying mechanical stress-induced activation. EMBO Rep. 2008;9(2):179-86. [PubMed: 18202720]

90. Newman S, Road J, Bellemare F, Clozel JP, Lavigne CM, Grassino A. Respiratory muscle length measured by sonomicrometry. Journal of applied physiology: respiratory, environmental and exercise physiology. 1984;56(3):753-64.

91. Boriek AM, Hwang W, Trinh L, Rodarte JR. Shape and tension distribution of the active canine diaphragm. Am J Physiol Regul Integr Comp Physiol. 2005;288(4):R1021-7. [PubMed: 15793029]

92. Boriek AM, Rodarte JR, Reid MB. Shape and tension distribution of the passive rat diaphragm. Am J Physiol Regul Integr Comp Physiol. 2001;280(1):R33-41. [PubMed: 11124131]

93. Sigurta A, Zambelli V, Bellani G. Renin-angiotensin system in ventilator-induced diaphragmatic dysfunction: Potential protective role of Angiotensin (1-7). Medical hypotheses. 2016;94:132-7. [PubMed: 27515219]

94. Smuder AJ, Min K, Hudson MB, et al. Endurance exercise attenuates ventilator-induced diaphragm dysfunction. J Appl Physiol. 2012;112(3):501-10.. [PubMed: 22074717]

95. Grosu HB, Lee YI, Lee J, Eden E, Eikermann M, Rose K. Diaphragm Muscle Thinning in Mechanically Ventilated Patients. Chest. 2012;142(5):1455-60. [PubMed: 23364680] 


\section{Key points}

- The renin-angiotensin system plays a key role in the control of blood pressure and fluid homeostasis.

- Growing evidence also reveals that hyperactivity of the renin-angiotensin system contributes to skeletal muscle wasting due to heart failure, kidney disease, and prolonged periods of mechanical ventilation.

- Several therapeutic approaches exist to prevent renin-angiotensin systeminduced muscle wasting; these include pharmacological blockade of angiotensin II receptors (type I) and preconditioning of skeletal muscle by endurance exercise training. 


\section{Classical Pathway}

Angiotensinogen

\section{Non-Classical Pathway} Renin Angiotensin ACE Angiotensin II<smiles>C1C2CC12</smiles>
ACE2 Angiotensin 1-9
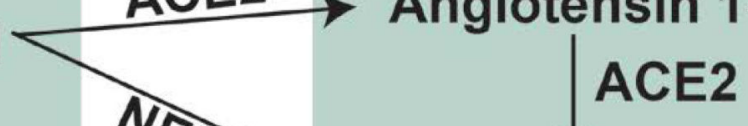

$A T_{1} R$

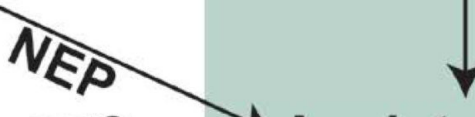

ACE2 $\longrightarrow$ Angiotensin 1-7

JAK kinase

STAT

Free calcium

NADPH oxidase

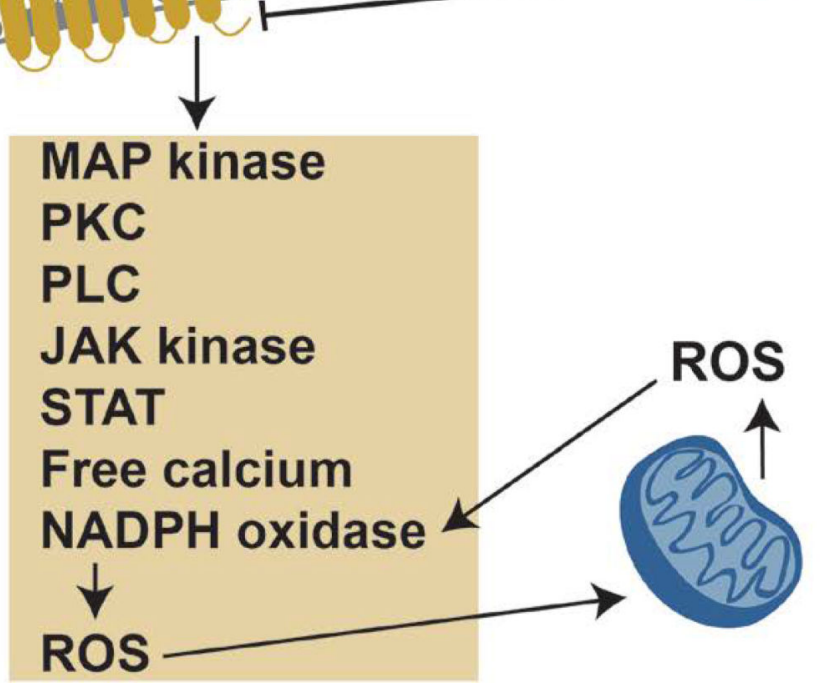

Figure 1.

Illustration of the classical and non-classical signaling pathways involved in the reninangiotensin system. See text for details. 


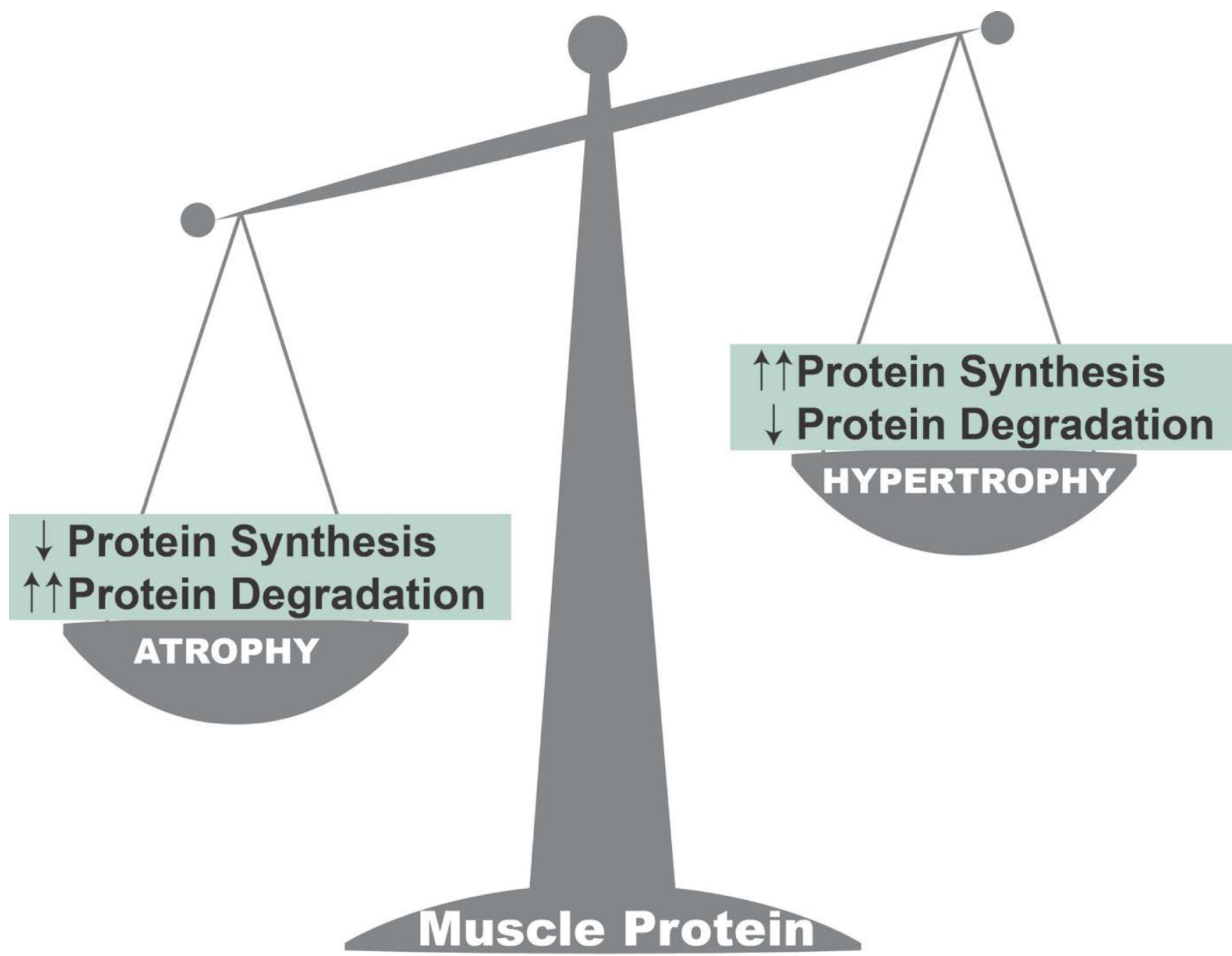

Figure 2.

The abundance of protein in skeletal muscle fibers is determined by a balance between the rates of protein synthesis and degradation. When the rate of protein degradation exceeds the rate of synthesis, there is a net loss of muscle protein and atrophy occurs. Conversely, when the rate of protein synthesis exceeds the rate of degradation, muscle growth (hypertrophy) occurs. 


\section{Chronic Heart Failure or Chronic Kidney Disease}

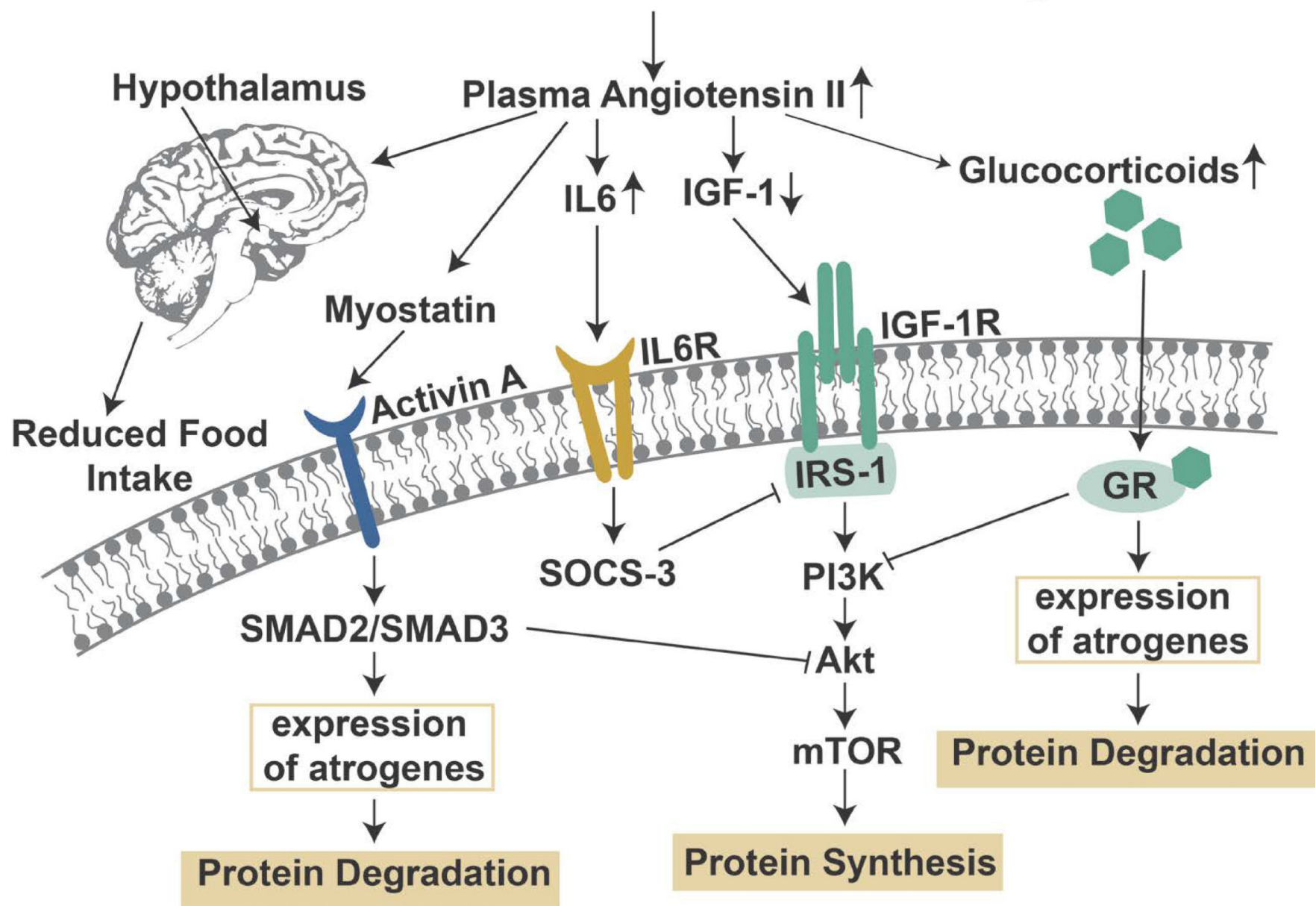

Figure 3.

This figure illustrates the molecules and signaling pathways involved in the indirect actions of Angiotensin II that promote skeletal muscle atrophy. See text for details. 


\section{Angiotensin II}

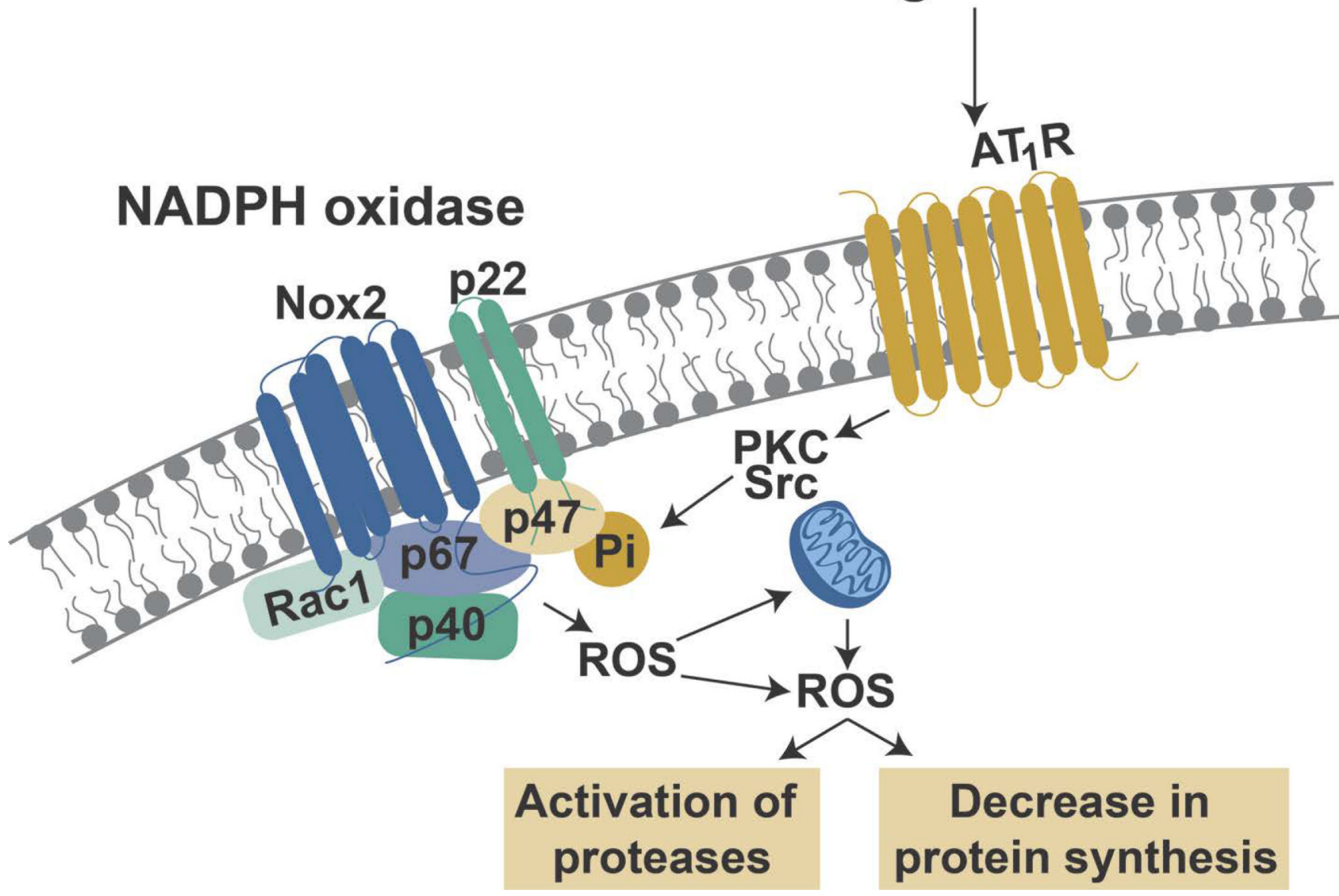

Figure 4.

Illustration of angiotensin receptor 1 (AT1R) signaling-induced activation of NADPH oxidase II (Nox2) and the resultant production of reactive oxygen species (ROS) and the potential cross-talk between Nox 2 and mitochondrial ROS production leading to the activation of proteases and a depression in muscle protein synthesis. See text for more details. 


\section{Prolonged MV promotes rapid diaphragmatic atrophy in humans}
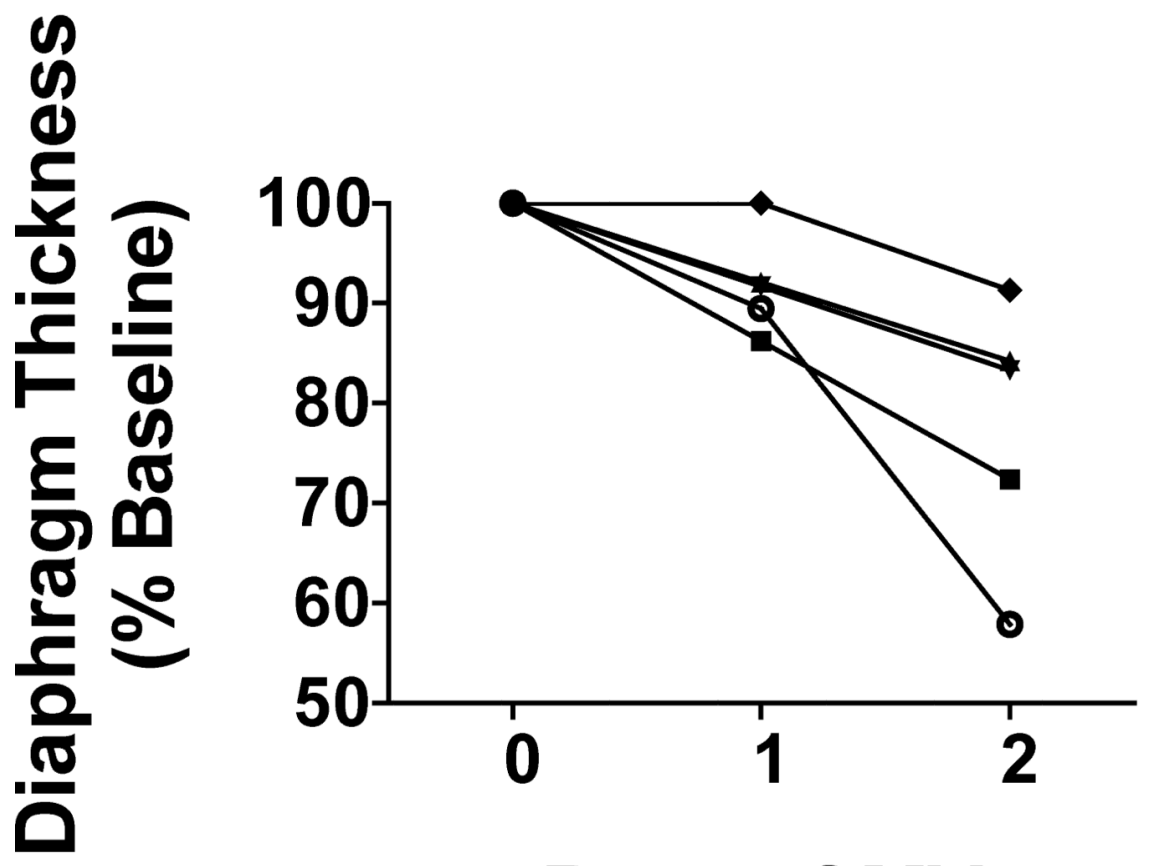

Days of MV

Figure 5.

Illustration of the rapid development of diaphragmatic atrophy in humans. Each line represents the time-related change in diaphragm thickness in one patient during three consecutive days of mechanical ventilation. Data are from Grosu et al. (95). 